\title{
LVIII. On the influence of the physical conditions of a substance on its absorption of light
}

\section{G.H. Livens B.A.}

To cite this article: G.H. Livens B.A. (1912) LVIII. On the influence of the physical conditions of a substance on its absorption of light, Philosophical Magazine Series 6, 24:142, 623-628, DOI: 10.1080/14786441008637366

To link to this article: http://dx.doi.org/10.1080/14786441008637366

册 Published online: 08 Jun 2010.

Submit your article to this journal \lceil

Џll Article views: 2

Q View related articles $\square$

Citing articles: 1 View citing articles 5 
contained helium at a pressure of about 8 millimetres. Such an active plate causes a vigorous recoil of the atoms of actinium D. Some throws were observed large compared with those of the a particle ; but the interpretation of the experiments is complicated by the natural disturbances due to the uncovered opening and to the strong ionization produced by the radiating source outside the opening. Further experiments are in progress to test whether this method can be employed to count the recoil atoms with certainty.

University of Manchester, August 16, 1912.

LVIII. On the Influence of the Physical Conditions of a Sultstance on its Absorption of Light. By G. H. Livens, B.A., Fellow of Jesus College, Cambridge *.

$\mathrm{T}$ $\mathrm{N}$ a recent communication to this Journal I have given a discussion of the effect of the density of a gas on its line spectrum. The results there obtained have, however, a wider application than appeared at first sight. An explanation of several other experimental facts concerning the effect of the physical circumstances of a substance on its absorption of light also appear to be contained in the theory. An interesting account of the phenomena under discussion is given by Prof. Wood in his book on 'Physical Optics.'

A few of the results here obtained have already been deduced by Dr. Havelock $\dagger$, and the reader is referred to that author's papers for a more complete discussion. Dr. Havelock's results however, are, owing to their generality, exhibited in rather a complicated form, and it seems as well to present them in the much simpler form in which they are deducible from the present theory.

1. The infuence of the nature of the solvent upon the position of the absorption lands of the dissolved substance.Wood gives a short account of Kundt's work on this subject, and summarizes the results in the following statement of Kundt's law:-If one transparent medium has a higher refractive index than another, the absorption bands of a dissolved substance lie nearer the red end of the spectrum when it is dissolved in the former than when it is dissolved in the latter.

I shall now adopt the notation of my previous paper and

* Communicated by the Author.

$\uparrow$ Proc. R. S. 19!0, 1911. 
quote the results there deduced. The equation of motion of the typical electron of mass $m$ and charge $e$ was taken in the form

$$
m \ddot{x}+h \dot{x}+k x=e\left(\mathrm{E}_{x}+a \mathrm{P}_{x}\right),
$$

where, $\mathrm{E}_{x}$ is the $x$-component of the electric force and $\mathrm{P}_{x}$ that of the polarization intensity. It was then found in the usual way that the absorption coefficient for light of a frequency $\left(n_{0}+\xi\right)$ was

$$
\mu k=\frac{1}{2} \frac{\rho_{1} n_{0} n_{1}^{\prime}}{\left[(1-a \mathrm{~A}) 2 n_{0} \xi+a \rho_{1}\right]^{2}+(1-a \mathrm{~A})^{2} n_{0}{ }^{2} n_{1}{ }^{\prime 2}},
$$

where, if $N_{1}$ is the number of electrons with the natural free period $n=n_{0}$,

$$
\rho_{1}=\frac{\mathrm{N}_{1} e^{2}}{m}, \quad n_{1}^{\prime}=\frac{h_{1}}{m}
$$

and

$$
\mathrm{A}=\Sigma \frac{e^{2}}{k-m n_{0}^{2}}
$$

this sum being taken over all the electrons per unit volume which have not the natural free period $n=n_{0}$.

From this we deduce at once that the maximum absorption is of amount

$$
\mu k=\frac{1}{2} \frac{\rho_{1}}{n_{0} n_{1}^{\prime}(1-a \mathrm{~A})^{2}}, \quad \cdot \quad, \quad . \quad .
$$

and occurs not in the position $\xi=0\left(n=n_{0}\right)$ but displaced towards the red end of the spectrum through a distance given by

$$
\xi=\frac{\pi \rho_{1}}{2(1-a \mathrm{~A}) n_{0}} .
$$

Now consider for simplicity the case when the spectrum of the dissolved substance consists only of a single line corresponding to the frequency $n=n_{0}$, and that this line does not occur in the absorption spectrum. The value of $\mathrm{A}$ then arises entirely from the electrons in the solvent. Moreover, if $\mu$ is the index of refraction of the solvent for the particular light of frequency $n=n_{0}$, then

$$
\begin{aligned}
\mu^{2} & =1+\frac{\mathrm{A}}{1-a \mathrm{~A}} \\
& =\left(1-\frac{1}{a}\right)+\frac{1}{a} \cdot \frac{1}{1-a \mathrm{~A}} .
\end{aligned}
$$

Thus an increase in $\mu$ arises mainly from an increase in the term $1 /(1-a \mathrm{~A})$. That is, an increase in $\mu$ corresponds 
Conditions of a Substance on its Alsorption of Light. $\quad 625$

to an increase in the displacement of the absorption lines towards the region of longer wave-length. This is Kundt's result.

Also according to Wood there appears to be evidence that the position of an absorption band depends on the concentration of the solution. 'This, of course, is exactly analogous to the case discussed in the previous paper. An increase in the density of the absorbing electrons displaces the line proportionately to the red end of the spectrum.

2. Absorption in a gas.-It is generally assumed that the absorption produced by a given quantity of gas is independent of its density. The important discovery was, however, made by Angström that the compression of a gas increased the intensity of its absorption, the mass of gas traversed remaining the same. The general results of this and much subsequent work of a similar kind are summarized by Wood in the four following statements :-

1. Beer's law does not in general hold for gases.

2. If to a gas at a definite volume we add a foreign gas which does not act on it chemically, its absorption is increased.

3. The absorption of a gas mixture is greater than the sum of the absorptions of its separate constituents each one taken at its partial pressure.

4. The absorption of a gas mixture is, on the contrary, equal to the sum of the absorptions of its constituent parts if each part is taken at a pressure equal to the total pressure.

Now equation (i.) above shows that the maximum absorption coefficient is

$$
\mu k=\frac{\rho_{1}}{2 n_{0} n_{1}^{\prime}} \cdot \frac{1}{(1-a \mathrm{~A})^{2}}
$$

and results (1) and (2) above are direct deductions from this formula. The simple statement contained in (4) does not appear, however, to be exact; although it is probably approximately true for the case of gases.

It is important to notice that the results of this and the last paragraph are simply two aspects of the one general problem. In the case of liquids the point investigated appears to have been the displacement of the absorption lines, their intensities being left out of consideration. In the case of gases it appears to have been the alterution in the intensity which first attracted notice. The two phenomena should, however, be present in both experiments, and the theory is equally valid for the two cases. 
3. On the broadening of the lines of a spectrum with increasing density.

We shall now turn to the last type of effect which is generally concerned with the alteration in the appearance of the lines or bands in a spectrum produced by changing the physical conditions. It is to be noticed that the theory here given will apply equally well to both emission and absorption spectra.

The theory in its present form does not, however, offer any explanation of a phenomenon of this kind; but an obvious and fairly plausible extension makes most of the experimental results so obvious that, on these grounds alone, it might be claimed as the effectively correct explanation. The extension is arrived at by a reconsideration of the constant $a$ which occurs in our formulæ. It was introduced as the coefficient of the polarization in the expression for the force on unit charge in a small cavity in the polarized medium. This expression represents simply the local part of the total forcive acting on a charge placed there, and arises entirely from the molecues in the immediate neighbourhood. Such a quantity, of course, is quite indeterminate in any real case, but an ideal estimate gives $a=1 / 3$. In an actual case the constant would approximate more or less accurately to this limiting value. Now in discussing spectrum lines in the above manner, we have to deal separately with the oscillations of each individual electron, and a value of this constant $a$ has to be used for each one. Now it seems most probable that the constant $a$ is not a constant for each individual electron but varies from one electron to another. It seems in fact that it would be better to take a range of more or less probable values grouped about the ideal value $a=1 / 3$. Moreover, the actual facts seem to indicate that the extent of the range should decrease as the condition of the substance approaches the limiting condition, $i$. e. the ideal limit of gas theory (practically when the density $\delta=0$ ). Moreover, the range cannot extend beyond the value $a=0$ on the one side; but except for the conditions implied in the analytical approximations, there seems to be $\mathrm{no}$ reason to exclude indefinite extension on the other side. Without attaching any special significance to the form of the functions given, I shall, for the sake of argument, assume that the range of most probable (not necessarily equally probable) values is bounded by the values

$$
a=\frac{1}{3} \cdot \frac{1}{1+c_{1} \delta}, \quad a=\frac{1}{3}\left(1+c_{2} \delta\right),
$$

where $c_{1}$ and $c_{2}$ are constants of each line and $\delta$ the density. 
Contitions of a Substance on its Alsorption of Light.

Under these assumptions the single line in the "ideal" spectrum of the substance corresponding to a frequency $n=n_{0}$ would be broadened out into a band whose brightest part would lie between the two positions at distances to the red side of the position $n=n_{0}$,

$$
\begin{aligned}
& \xi_{1}=\frac{\rho_{1}}{2 n_{0}} \cdot \frac{1}{1+\left(1+c_{1} \delta\right)\left(1+c_{2} \delta\right)}, \\
& \xi_{2}=\frac{\rho_{1}}{2 n_{0}} \cdot \frac{\left(1+c_{2} \delta\right)\left(1+c_{1} \delta\right)}{1+\left(1+c_{1} \delta\right)\left(1+c_{2} \delta\right)} .
\end{aligned}
$$

If we assumed that each value of $a$ between the above limits were equally probable for any one electron, the portion of the band between $\xi_{1}$ and $\xi_{2}$ would be uniformly bright. Tnequalities in the probability of the occurrence of the various values of $a$, which it seems more reasonable to assume, would account for differences in the intensity of the various parts of the band, the brighter parts corresponding to the more probable values of $a$. A simple explanation of the constitution of channelled spectra seems to be here involved; but this point is reserved for future consideration.

The value of $\rho_{1}$ in the above formulx is also proportional to the density of the substance, and so we can write

$$
\begin{aligned}
& \xi_{1}=\xi_{0} \frac{\delta}{1+\left(1+c_{1} \delta\right)\left(1+c_{2} \delta\right)^{\prime}} \\
& \xi_{2}=\xi_{0} \frac{\delta\left(1+c_{1} \delta\right)\left(1+c_{2} \delta\right)}{1+\left(1+c_{1} \delta\right)\left(1+c_{2} \delta\right)} ;
\end{aligned}
$$

and therefore the breadth of the band is

$$
\xi_{2}-\xi_{1}=\xi_{0} \delta \frac{\left(1+c_{1} \delta\right)\left(1+c_{2} \delta\right)-1}{\left(1+c_{1} \delta\right)\left(1+c_{2} \delta\right)+1} .
$$

This result enables us to deduce at least a qualitative explanation of the effect of an increase in density on the lines of the spectrum. The broadening out of the line is always much faster towards the side of longer wave-length. In fact the broadening towards the violet side, although practicaliy symmetrical with that on the other side when the density is small, soon becomes very slow. This explains the unsymmetrical broadening of most of the lines of ordinary spectra. An explanation of some of the remarkable phenomena discovered by Wood particularly in the mercury line 2536 is also involved.

The extension of the above ideas to include the action of a foreign chemically inactive gas is of course obvious. The 
theoretical conclusions derived agree with the experimental results given by Wood.

This theory also provides for the broadening of the lines in Humphrey's experiments on the "pressure shift." There is, however, a still more obvious reason for taking a range of values of the constant $a$ as above. The density of the gas varies considerably from the cooler parts in the surrounding air to the very hot portions in the centre of the arc.

It thus appears that by taking advantage of the latitude allowed by the theoretical uncertainty in the value of the constant $a$, we can explain a large number of the complications present in ordinary spectra. We have merely to avoid mutually inconsistent hypotheses in explaining the var ous types of the phenomena. Any consistent and reasonable set of properties for the constant $a$ would appear to provide a theoretically effective explanation of the phenomena under discussion. We might regard the experimental results as providing the necessary laws for the variation of the constant $a$ with the physical conditions of the material. It is, however, important to notice that the more extensive list of physical properties would involve only the mean value of the constant $a$ taken for all the electrons of the substance. Such values are deducible from known properties of the substance in bulk. Spectrum analysis alone can separate out the possibly different values of $a$ with which we are concerned when discussing the individual electrons.

Dr. Havelock has given a discussion of the dependence of the mean value of the constant $a$ on the physical conditions of the substance.

The theory here given is not, however, complete. It appears to fail in one very important respect. Although in the majority of cases the actual facts are as stated, yet there are some important exceptions. For instance, the displacement of certain absorption and emission lines in the spectra of not uncommon substances is not towards the red end of the spectrum, but towards the violet end. The associated phenomena of asymmetrical broadening also frequently takes place in this direction. The theory here developed appears, at present, quite unable to admit of an explanation of these divergences from what appears, after all, to be the general rule which it does so well explain. Further investigation may, however, confirm the results already deduced : and we must then look for the loophole which will enable us to extend the theory to the above anomalies. 